\title{
Mycoplasma Pneumonia with Cold Agglutinin Autoimmune Hemolytic Anemia: Analytical Discrepancies and Spurious Results-A Rare Case Report
}

\author{
${ }^{1}$ Majed AB Momin, ${ }^{2} \mathrm{KM}$ Reddy, ${ }^{3} \mathrm{~B}$ Saroj K Prusty
}

\begin{abstract}
Aim: To highlight the discrepancies in hemogram parameters and blood grouping related to cold agglutinin hemolytic anemia cases which not only helps to the diagnosed underlying pathogen but prevents its complication.

Background: Cold agglutinin hemolytic anemia is a rare form of autoimmune hemolytic anemia caused by cold-reacting autoantibodies. The automated hematology analyzer hemogram analysis of these patients sample is related to analytical discrepancies and spurious result. Immunohematology workup including peripheral smear findings, warming the ethylenediamine-tetra-acetic acid (EDTA) sample and cold agglutinin titer helps to resolve such discrepancies and gives a diagnostic clue of rare presentation of atypical pneumonia which responds to specific antibiotics.
\end{abstract}

Case report: A 55-year old male presented with fever, shortness of breath and cough for two weeks. A blood sample drawn for hemogram show high mean cell hemoglobin $(\mathrm{MCH})$, mean corpuscular hemoglobin concentration $(\mathrm{MCHC})$ and low red blood cells (RBCs) count and hematocrit with discrepancies in blood groupings. Peripheral blood film show significant autoagglutination of RBCs and infective changes in white blood cell morphology suggest cold agglutinin secondary to mycoplasma pneumonia. Further immunohematology workup and serological investigation confirm cold agglutinin autoimmune hemolytic anemia with mycoplasma pneumonia.

Conclusion and clinical significance: This case report emphasizes blood count analytical and blood grouping problems related to cold agglutinins and also sensitize to search underlying etiology like mycoplasma pneumonia to start specific antibiotics and to reduce morbidity and mortality.

Keywords: Analytical discrepancies, Cold agglutinin, Management, Mycoplasma pneumonia.

How to cite this article: Momin MAB, Reddy KM, Prusty BSK. Mycoplasma Pneumonia with Cold Agglutinin Autoimmune HemoIytic Anemia: Analytical Discrepancies and Spurious Results-A Rare Case Report. Journal of Postgraduate Medicine, Education and Research, Oct-Dec 2018;52(4):180-182.

Source of Support: Nil

Conflict of Interest: None

\footnotetext{
${ }^{1}$ Consultant Pathologist, ${ }^{2}$ Consultant, ${ }^{3}$ Consultant Intensivist

${ }^{1-3}$ Department of Laboratory Medicine, Yashoda Hospitals Malakpet, Hyderabad, India

Corresponding Author: Majed AB Momin, Consultant Pathologist, Department of Laboratory Medicine, Yashoda Hospitals Malakpet, Hyderabad, India, Phone: +919849076574, e-mail: majedmomin878@yahoo.co.in
}

\section{BACKGROUND}

Mycoplasma pneumonia infections with respiratory tract involvement are generally asymptomatic. However, 3 to $10 \%$ of patients develop clinical pneumonia. Extrapulmonary complications seen in $25 \%$ cases, before, during, after or in absence of pulmonary sign. ${ }^{1}$

Autoimmune hemolytic anemia (AIHA) is classified as warm and cold AIHA. In cold AIHA, the hemolysis is mediated by $\operatorname{IgM}$ antibodies directed against red cells. The term cold refers to the fact that antibody binds maximally at a temperature below $37^{\circ} \mathrm{C}$. The most efficient temperature for binding is called thermal amplitude. After binding cold agglutinin causes agglutination of erythrocytes and antigen-antibody complex induces complement-mediated hemolysis. ${ }^{2}$ Cold agglutinin interferes with the analysis of RBCs and its related parameters (hematocrit, $\mathrm{MCV}, \mathrm{MCHC}$ ). However, hemoglobin, leucocyte count and platelets are not affected. ${ }^{3}$

In the present case report, we evaluated cold agglutinins as hematological analyzer discrepancies and diagnose underlying etiology which was sensitized to a particular group of antibiotics.

\section{CASE REPORT}

A 55-year old male presented to the emergency room with symptoms of fever, shortness of breath and cough for two weeks. On physical examination patient appeared restless, febrile (100.3f), tachypnic (24 breaths/min) and tachycardia (108 beats/min). Blood pressure was 130/80 $\mathrm{mm} \mathrm{Hg}$. On respiratory examination, bilateral basal crepitations heard. Another systemic examination was unremarkable.

The EDTA sample shows visible agglutination at the wall of the tube (Fig. 1A green arrow), and a peripheral smear prepared from the same sample show granular deposit (Fig. 1A red arrow). Hemogram showed mild anemia, low hematocrit, high $\mathrm{MCH}, \mathrm{MCHC}$, suggestive spurious macrocytosis (Fig. 1C), white blood cells and platelets were within normal limits. The same sample after $37^{\circ}$ incubation for 10 minutes show normal hematocrit for hemoglobin and normal RBCs indices (Fig. 1D). Peripheral smear Leishman stain microscopy show 


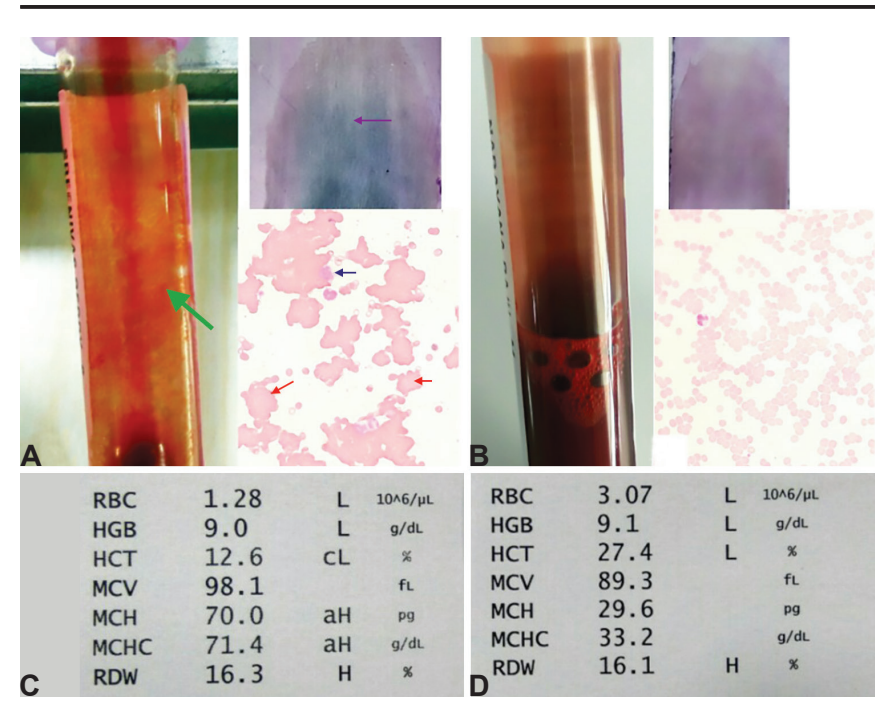

Figs 1A to D: (A) Visible agglutination in vacutainer (green arrow), granular stain (red) and microscopic autoagglutination (red), (B) After warming no agglutination in vacutainer and peripheral smear microscopy, (C) Hemogram before warming sample (low RBCs count, hematocrit and high indices), (D) After warming correction of RBCs count, hematocrit and RBCs indices

Significant agglutination (Fig. 1A) and neutrophils show cytoplasmic vacuolation. Same sample after 37 degree incubation for 10 minutes show normal hematocrit for hemoglobin and normal RBCs indices (Fig. 1D). Post incubation leishman stained film show no agglutination (Fig. 1B).

Biochemical parameters, like serum creatinine $2.2 \mathrm{mg} / \mathrm{dL}$ ( 0.8 to $1.5 \mathrm{mg} / \mathrm{dL}$ ) blood urea $112 \mathrm{mg} / \mathrm{dL}$ (19 to $43 \mathrm{mg} / \mathrm{dL}$ ), S. bilirubin $4 \mathrm{mg} / \mathrm{dL}(0.2-1.3 \mathrm{mg} / \mathrm{dL})$ with conjugated 0.8 $\mathrm{mg} / \mathrm{dL}$ and unconjugated $3.2 \mathrm{mg} / \mathrm{dL}$, elevated LDH 744 $\mathrm{u} / \mathrm{L}$ (120 to $246 \mathrm{U} / \mathrm{L})$. Urine examination reveals proteinuria. Serum electrolytes were within normal limits. Serological examination for human immuno deficiency virus (HIV), Hepatitis B surface antigen (HBsAg), and hepatitis $\mathrm{C}$ virus (HCV) are nonreactive. Other tests for malaria, brucella, and typhoid are negative.

Erythrocyte sedimentation rate was high $110 \mathrm{~mm} /$ hour. Coagulation studies were within normal limits. Reticulocyte count was $6 \%$. Forward grouping in tube showed $\mathrm{AB}$ positive, but weak agglutination with anti-A antisera. Forward grouping was again performed by tube method after proper washing of cells with warm saline showed no reaction with anti-A antisera, but $4+$ reaction was noted with anti-B, anti-AB, anti-D antisera, and the group was $B$ positive. Reverse grouping showed a reaction with A cells, so B group was confirmed. The direct Coombs' test was positive $(4+)$ and the differential card test show positivity for C3b, C3d with negative for IgG. Patient cell and serum were incubated at $4^{\circ}, 18^{\circ}, 22^{\circ}$ at room temperatures and $37^{\circ} \mathrm{C}$ in an incubator for a period of 30 minutes to confirm further cold antibodies in the patient blood. The auto-control showed $4+$ reaction at $40^{\circ} \mathrm{C}$, weaker reaction at $18^{\circ} \mathrm{C}$, but no reaction was noted

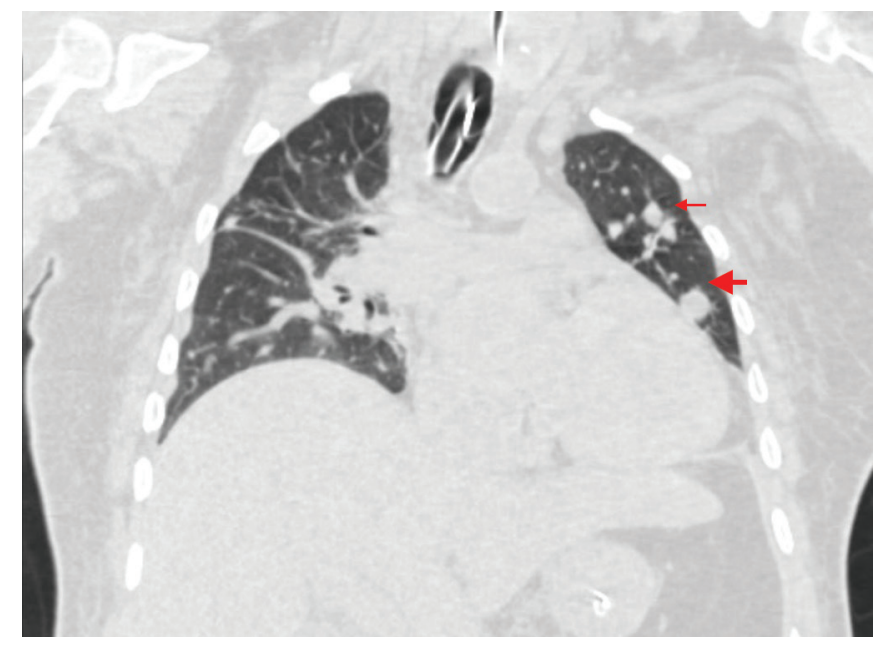

Fig. 2: CT image (coronal view) with nodular infiltrates (red arrow)

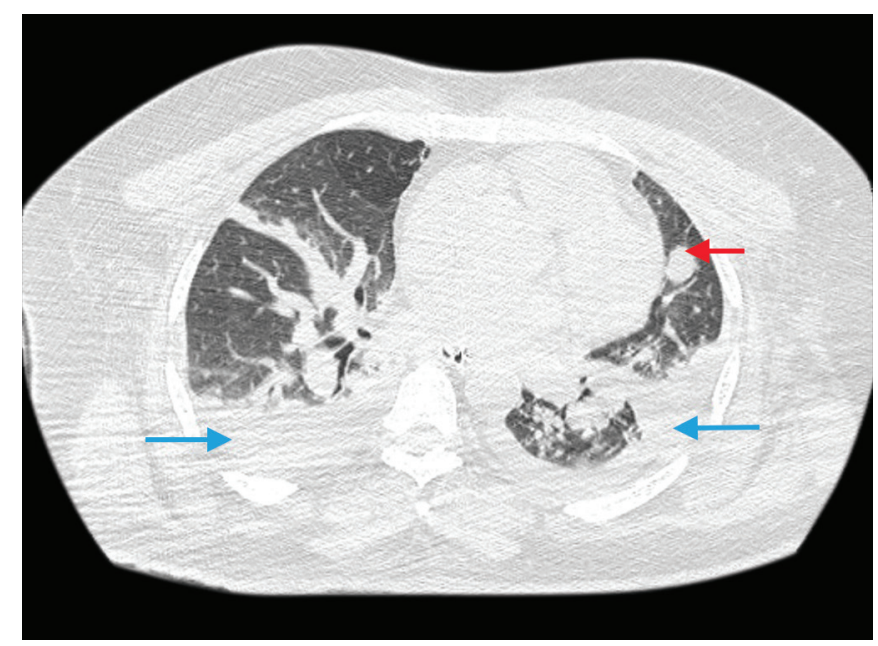

Fig. 3: CT image (axial view) with bilateral consolidation (blue arrow) and nodular infiltrates (red arrow)

in the tubes incubated at $22^{\circ}$ and $37^{\circ} \mathrm{C}$. The titration of cold antibodies done by double dilution technique, and the titer was 1 in 64 shows the benign nature of the antibodies. Serological test for atypical pneumonia panel came positive for mycoplasma pneumonia (serum EIA).

Chest X-ray showed bilateral opacities. CT scan chest showed consolidation with few nodular infiltrates in both lungs and mild pleural reaction on both sides (Figs 2 and 3).

Based on the clinical, hematological and serological investigation, cold agglutinin autoimmune hemolytic anemia as a complication of mycoplasma pneumonia and acute tubular necrosis was considered and treated with macrolides antibiotics.

\section{DISCUSSION}

Mycoplasma pneumonia is a common cause of community-acquired pneumonia internationally, and in India, it accounts for 25 to $35 \%$ of all outpatient pneumonia. Its an airborne transmission via respiratory 
droplets with an incubation time of 7 days to 3 weeks. ${ }^{4}$ Clinical presentation are nonspecific and include a dry or a productive cough, fever, headache, and malaise.

Multiorgan involvement is unusual. Other nonspecific complications include gastrointestinal, glomerulonephritis and hemolytic anemia. The suggested pathogenesis behind mycoplasma pneumonia and hemolytic anemia is the cross antigenicity between mycoplasma and I antigen present in red cell membrane. ${ }^{4}$

The cold agglutinins are seen in mycoplasma pneumonia in $50 \%$ of cases. Cold agglutinins are import general manifest (IgM) molecule which appears in blood one week after the onset of illness and decline after 2 to 6 weeks. Cold agglutinins are more specific for I antigen on red blood cells and after attaching initiate complement pathway (classical), and as the blood returns to the warmer temperatures within the body, cold agglutinin dissociates from the red cell membrane, but complement activation continues. It is the anti-C $3 \mathrm{~d}$ component of polyspecific AHG that accounts for the positive direct coombs test (DCT) and the indirect Coombs test, was negative as seen in the present case. Antibody-mediated hemolysis seen in $10 \%$ patient with mycoplasma pneumonia infections and manifested as reticulocytosis, increased in unconjugated bilirubin. Increased in lactate dehydrogenase with decreased level haptoglobin. ${ }^{5}$

The analysis of specimen show visible agglutination at the wall of EDTA anticoagulant vacutainer. An automated hematology analyzer show underestimation of RBCs count, hematocrit count while spurious increased in $\mathrm{MCH}$ and $\mathrm{MCHC}$, seen in the histogram and few cases, it shows cross linearity of colter analyzer. The micro aggregates of RBCs are counted as single cells, and large clumps of cells are excluded from the count which leads to decreased RBC count. These abnormal RBCs count affect hematocrit, $\mathrm{MCH}$, and $\mathrm{MCHC}$. These discrepancies and spurious result are corrected by warming a sample, which corrects not only hemogram values but also agglutination seen in peripheral smear. ${ }^{3,6}$

Cold agglutinins may also be seen in other infectious diseases like infectious mononucleosis and neoplastic disease like lymphoproliferative disorder. Clinical clue includes acrocyanosis and Raynaud's phenomenon while spurious lab finding with autoagglutination corrected after warming samples indicates underlying cold agglutinins.

Mycoplasma organism is fastidious and difficult to grow in culture and diagnosis is based on serological or polymerase chain reaction (PCR) gene amplification techniques. ${ }^{7}$ Mycoplasma lacks a cell wall and therefore resistant to antibiotics like beta-lactam antimicrobials, and the drug of choice is macrolides (azithromycin/ clarithromycin) as they directed at inhibition of deoxyribonucleic acid (DNA) or protein synthesis. Furthermore, in the setting of discrepancies related blood groupings and possible hemolysis use of packed red cell transfusion should be limited. ${ }^{8}$

\section{CONCLUSION}

To conclude, cold agglutinin hemolytic anemia should be considered in any patient presenting with pneumonia and hemolysis. Lab technologist and pathologist should be aware of these discrepancies in RBCs, related parameters in automated hematology analyzer and blood groupings secondary to the presence of cold agglutinin. Detection of cold agglutinin not only helps to find out underlying etiology but the management of disease as mycoplasma lacks cell wall which renders antimicrobial ineffective except macrolides.

\section{ACKNOWLEDGMENT}

Authors would like to appreciate the technical staff of the hematopathology department.

\section{REFERENCES}

1. Clyde WA Jr. Clinical overview of typical Mycoplasma pneumoniae infections. Clin Infect Dis 1993 Aug;17:(Suppl 1): S32-S36.

2. Gehrs BC, Friedberg RC. Autoimmune hemolytic anemia. Am J Hematol 2002;69:258-271.

3. Kakkar N. Spurious automated red cell parameters due to cold agglutinins: a report of two cases. Indian J Pathol Microbiol 2004 April-June;47:250-252.

4. Dey AB, Chaudhry R, Kumar P, Nisar N, Nagarkar KM. Mycoplasma pneumoniae and community-acquired pneumonia. Natl Med J India. Mar-Apr; 2000:13(2):66-70.

5. Duffy TP. Autoimmune hemolytic anemia and paroxysmal nocturnal hemoglobinuria. In: Simon TL, Synder EL, Solheim BG, Stowell CP, Strauss RG, Petrides M, editors. Rossi's principles of transfusion medicine. 4th ed. AABB: Wiley-Blackwell, March; 321-331.

6. Solanki DL, Blackburn BC. Spurious red blood cell parameters due to serum cold agglutinins: observations on Ortho ELT-8 Cell counter. Am J Clin Pathol 1985; Feb;83:218-222.

7. Kraft M, Cassell GH, Henson JE, Watson H, Williamson J, Marmion BP et al. Detection of Mycoplasma pneumoniae in the airways of adults with chronic asthma. Am J Respir Crit Care Med. 1998 Sep;158(3):998-1001.

8. Suzuki S, Yamazaki T, Narita M, Okazaki N, Suzuki I, Andoh $\mathrm{T}$ et al. Clinical evaluation of macrolide-resistant Mycoplasma pneumoniae. Antimicrob Agents Chemother. 2006 Feb;50(2): 709-712. 